\title{
Stoichiometric analysis of superficial Ba doped Strontium Titanium Oxide layers using APT: the case of the missing Oxygen!
}

Richard J. H. Morris ${ }^{1}$, Mihaela Popovici ${ }^{2}$, Johan Meersschaut ${ }^{3}$, Jeroen Scheerder ${ }^{1}$, Ludovic Goux ${ }^{2}$, Gouri Kar $^{2}$, Claudia Fleischmann ${ }^{4}$, Wilfried Vandervorst ${ }^{5}$ and Paul van der Heide ${ }^{3}$

${ }^{1}$ Imec, Kapeldreef 75, 3001 Leuven, Belgium, Leuven, Vlaams-Brabant, Belgium, ${ }^{2}$ IMEC, United States, ${ }^{3}$ Imec, Kapeldreef 75, 3001 Leuven, Belgium, United States, ${ }^{4}$ Imec, Kapeldreef 75, 3001 Leuven, Belgium,Quantum solid-state physics group, KU Leuven, Celestijnenlaan 200D, 3001 Leuven, Belgium, Belgium, ${ }^{5}$ Imec, Kapeldreef 75, 3001 Leuven, Belgium, Quantum solid-state physics group, KU Leuven, Celestijnenlaan 200D, 3001 Leuven, Belgium, United States

Oxides play a significant role in the semiconductor field. This includes $\mathrm{SiO} 2$ through to $\mathrm{HfO} 2$ for insulating gate layers [1]; to the more complex transition-metal oxide perovskites (e.g. SrTiO3, $\mathrm{BiFeO} 3$ ) [2] which are now being explored for future memory and quantum computing applications. Substoichiometric oxygen has been shown to increase the leakage current while accurate characterization of these layers is challenging but remains a crucial step in their evolutionary process. Given their scale (a few $\mathrm{nm}$ ) and evolving complexities, few techniques offer the spatial resolution combined with mass analysis capabilities required, hence atom probe tomography (APT) [3] is now being explored.

In this study, atomic layer deposited $(\sim 7 \mathrm{~nm})$ Ba doped SrTiO3 layers have been analyzed using APT (see figure 1). Additionally, Rutherford backscattering spectrometry (RBS) was used to determine the ratio between Ba:Sr:Ti and Time-of-Flight/Energy elastic recoil detection (ERD) the Sr:O ratio. The objective was to establish the correct layer stoichiometry, a critical parameter for tailoring device performance, from a single analysis. A LEAP $5000 \mathrm{XR}$ was used for the APT analysis of the sample, and from the mass spectra obtained, apparent layer stoichiometries determined. APT showed an excellent Ba:Sr:Ti ratio agreement to RBS (see Table I), however, from the APT Sr:O ratio compared with the ERD, a significant oxygen underestimation was observed.

In conclusion, given that APT is expected to have the same detection efficiency for all ions, it was found through comparison with RBS and ERD that the oxygen quantification in APT from Ba doped SrTiO3 layers was underestimated by $\sim 20$ at. \%. The excellent agreement in Ba:Sr:Ti ratio between APT and RBS would indicate that any potential loss mechanism(s) present has (have) an equivalent impact for all three elements. Correspondingly, the loss mechanism(s) is (are) either larger for oxygen, or there is an additional influence, which suppresses the generation/detection of the oxygen ions in APT. 


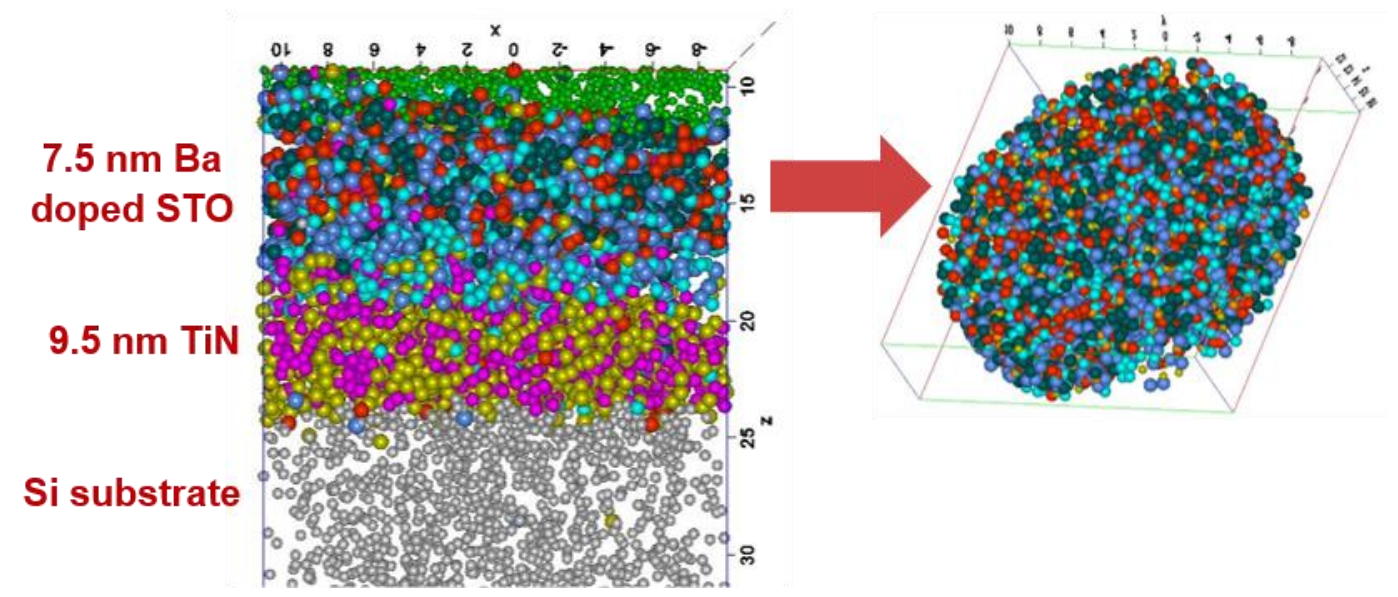

Figure 1. Figure 1. (a) Reconstruction of data taken from a thin film of Ba doped SrTiO3 on TiN and (b) The isolated $\mathrm{Ba}$ doped $\mathrm{SrTiO} 3$ region of interest used for the stoichiometric analysis.

\begin{tabular}{|c|c|c|}
\hline Element & APT & RBS \\
\hline & Element ratio & Element ratio \\
\hline $\mathrm{Ba}$ & 8.1 & 9.5 \\
\hline $\mathrm{Sr}$ & 30.0 & 28.4 \\
\hline $\mathrm{Ti}$ & 61.9 & 62.1 \\
\hline
\end{tabular}

Figure 2. Table I. The Ba:Sr:Ti ratio found from APT and RBS where an excellent agreement for APT with the well-established RBS technique is attained.

\section{References}

[1] Stephen Hall et al, J. Telecomm and Inform Tech, 2, 33, 2007.

[2] Dianxiang Ji et al, Nature, 570, 87, 2019.

[3] Tom Kelly \& Michael Miller, Rev. Sci. Instrum, 78, 031101, 2007. 